\title{
Preface I
}

\section{Prebiotic chemistry of phosphorus chemistry and origin of biomolecules}

It is a pleasure and honor for me to provide the preface for the book Phosphorus Chemistry: The Role of Phosphorus in Prebiotic Chemistry edited by Professor Yufen Zhao on the occasion of the 95th anniversary celebration of Xiamen University. There is no other group in the world better qualified to review the role of phosphorus in the prebiotic formation of essential biomolecules, such as amino acids, nucleosides, nucleotides, oligonucleotides and finally peptides as well as nucleic acids. This review is based on nearly 30 years of systematic research by Professor Zhao and her group on phosphorus and life, having identified phosphorylation as one of the key events in prebiotic evolution on the early Earth. $N$-phosphoryl amino acids play an essential role in this scenario. It was found that $\alpha$-amino acids after $\mathrm{N}$-phosphorylation could conduct a series of reactions important for the origin of life, such as peptide formation, ester exchanges, phosphoryl group migration, nucleotide formation, as well as membrane formation. It was demonstrated that only the $\alpha$-amino acids could be activated to form a peptide, while the $\beta$ - and $\gamma$-amino acid remained unchanged under the same conditions. Phosphoryl amino acid 5'-nucleosides having a $\mathrm{P}-\mathrm{N}$ bond have been described as a model of the origin of amino acid homochirality and the genetic code: the chiral selection of the earliest amino acids for l-enantiomers seemed to be determined by a stereochemical/physicochemical relationship. On the basis of this abundance of data on the role of phosphorylation in prebiotic processes, a comprehensive model of coevolution of proteins and nucleic acids in the origin of life scenario was formulated. The 11 chapters of this book provide an insight into this work on the importance of phosphorus for life, in prebiotic chemistry as well as in contemporary life.

Gerda Horneck

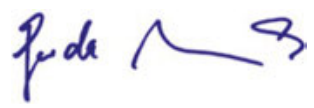

Former Deputy Director of the Institute of Aerospace Medicine, German Aerospace Center DLR, Cologne, Germany (retired)

Honorary President of the European Astrobiology Network Association EANA 
